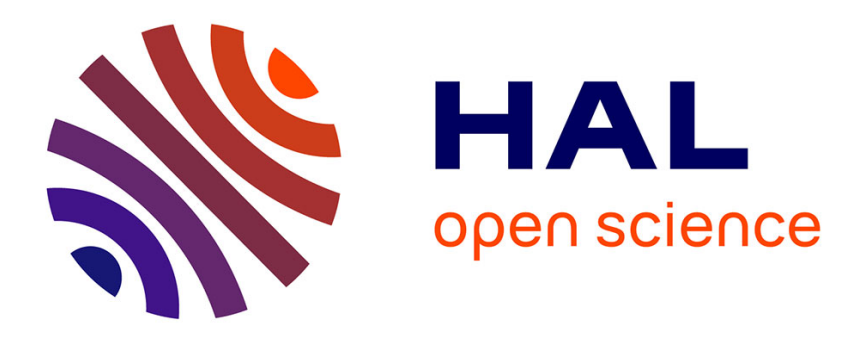

\title{
La politique étrangère entre l'Elysée et Matignon Samy Cohen
}

\section{To cite this version:}

Samy Cohen. La politique étrangère entre l'Elysée et Matignon. Politique étrangère, 1989, 54 (3), pp.487-503. 10.3406/polit.1989.3878 . hal-01009368

\section{HAL Id: hal-01009368 \\ https: / hal-sciencespo.archives-ouvertes.fr/hal-01009368}

Submitted on 17 Jun 2014

HAL is a multi-disciplinary open access archive for the deposit and dissemination of scientific research documents, whether they are published or not. The documents may come from teaching and research institutions in France or abroad, or from public or private research centers.
L'archive ouverte pluridisciplinaire HAL, est destinée au dépôt et à la diffusion de documents scientifiques de niveau recherche, publiés ou non, émanant des établissements d'enseignement et de recherche français ou étrangers, des laboratoires publics ou privés. 


\section{La politique étrangère entre l'Elysée et Matignon}

In: Politique étrangère $N^{\circ} 3$ - 1989 - 54e année pp. 487-503.

Citer ce document / Cite this document :

Cohen. La politique étrangère entre l'Elysée et Matignon. In: Politique étrangère $N^{\circ} 3$ - 1989 - 54e année pp. 487-503.

doi : 10.3406/polit.1989.3878

http://www.persee.fr/web/revues/home/prescript/article/polit_0032-342X_1989_num_54_3_3878 


\section{Abstract}

The Locus of Foreign Policy : Elysée or Matignon?, by Samy Cohen

Eighteen months after 8th May 1988, the question of cohabitation dropped out of sight as a public issue, although history could well repeat itself in 1993 and the problem arise once more of who should have control of foreign policy - the President or the Prime Minister. The Constitution provides no clear answer. The experience of cohabitation from 1986-88 was struggle for hegemony interspersed with épisodes of genuine harmony. In the end, the foreign policy process dependend little on the Constitution but was shared three ways according to the internai or external context and on whoever was the most nimble-footed protagonist in political, administrative or diplomatie manoeuvring. If the climate were favourable to the government, it might be able to force the Président to choose between submission to its will or resignation, unless the Constitution is changed to avoid this risk. Strangely none of the prominent players seem at ail anxious to learn from past expérience. In May 1988, ail trace of cohabitation was effaced and the President took over control of foreign policy more fully than during the years 1981-86.

\section{Résumé}

Dix-huit mois après le 8 mai 1988, la cohabitation disparaît du débat public. Pourtant l'histoire peut se répéter en 1993 et avec elle l'interrogation : qui du Président ou du premier ministre devra diriger la politique étrangère ? La Constitution ne fournit pas de réponse claire. La pratique des années 19861988 montre que la cohabitation a été une lutte pour l'hégémonie, entrecoupée de moments de réelle connivence. Elle a abouti à partager la politique étrangère en trois sphères d'influence qui doivent moins leur existence à la Constitution qu'au contexte intérieur et extérieur et à l'habileté de chacun des protagonistes à utiliser ses propres ressources politiques, administratives et diplomatiques. Dans une conjoncture politique avantageuse pour le gouvernement, celui-ci pourrait obliger le Président à choisir entre la résignation et la démission. A moins qu'intervienne d'ici là un aménagement de la Constitution qui écarterait ce danger. Etrangement, aucun des grands acteurs ne semble vouloir tirer les leçons du passé. Mai 1988 : toute trace de la cohabitation est effacée et la présidence reprend le contrôle de la politique étrangère. Plus entièrement que dans les années 1981-1986.

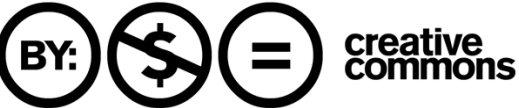




\section{\begin{tabular}{l|l} 
Samy COHEN * & $\begin{array}{l}\text { La politique étrangère } \\
\text { entre l'Elysée et Matignon }\end{array}$
\end{tabular}}

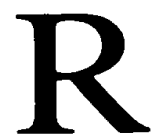

efoulée, reléguée au rang d'un accident de l'histoire : dix-huit mois après la victoire de François Mitterrand à l'élection présidentielle de mai 1988, la cohabitation disparaît du débat public, la page semble tournée. Il faut pourtant se rendre à l'évidence : à défaut d'un aménagement constitutionnel, une nouvelle période de cohabitation entre un président et un gouvernement de tendances politiques opposées est toujours possible. L'histoire peut même se répéter dès l'été 1993, échéance des prochaines élections législatives. Se reposera alors l'inévitable question : en politique étrangère, qui du Président ou du premier ministre devra gouverner?

Interrogation sans objet, rétorqueront les contemplateurs de la Constitution, puisque celle-ci définit les rôles de chacune des deux têtes de l'exécutif, fixe par avance les règles de la cohabitation. Que les futurs hôtes de l'Elysée et de Matignon s'engagent donc à la respecter tout comme François Mitterrand et Jacques Chirac l'avaient fait en 1986: "La Constitution, rien que la Constitution, toute la Constitution ", avait proclamé, on s'en souvient, le chef de l'Etat dans son message au Parlement, le 8 avril 1986. « Ni François Mitterrand, ni moi-même ne voulons pratiquer le coup d'Etat (...), l'un et l'autre nous respectons les règles de la démocratie et de la Constitution... », avait renchéri peu après Jacques Chirac [1].

Espérer que la Constitution serve de code de conduite relève de la naïveté sinon de l'aveuglement. Dans la pratique, le Président et le premier ministre se sont montrés fort peu respectueux du texte du 4 octobre 1958, les événements l'ont montré de manière accablante. Pouvait-il en être autrement? La Constitution ne laisse - si aucun des grands responsables nationaux ne s'efface devant l'autre - que le choix entre la dyarchie ou l'affrontement. Appliquée à la lettre, la Constitution conduit à un pur bicéphalisme tant les compétences sont enchevêtrées entre un Président, chef des armées (art. 15), garant de l'indépendance nationale et de l'intégrité du territoire (art. 5), dont la vocation est de négocier et de ratifier les traités (art. 52) et un gouvernement qui détermine et conduit la politique de la nation, dispose de la force armée (art. 20), tandis que le premier ministre est responsable de la défense nationale (art. 21).

* Chercheur au Centre d'études et de recherches internationales (CERI) de la Fondation nationale des sciences politiques, Paris. 
François Mitterrand et Jacques Chirac ont eu l'heureuse inspiration de rejeter cette méthode de gouvernement qui aurait considérablement affaibli la capacité d'initiative et de décision de la France. On imagine sans difficulté le coût d'un désaccord au sommet de cet exécutif à l'heure, voire à la minute, où la France aurait à se défendre par le déclenchement du feu atomique. François Mitterrand et Jacques Chirac choisiront donc la lutte pour la première place.

Chacun d'eux tentera de convaincre l'opinion que la Constitution lui confie la direction de la diplomatie. Bien avant le 16 mars 1986 , les intentions du futur gouvernement sont clairement affichées : il ne laissera pas au chef de l'Etat la haute main sur la politique étrangère dont on sait, en raison de l'imbrication des affaires extérieures et intérieures, qu'elle lui permettra d'exercer une influence sur le reste de la politique de la nation. Position qui attirera cette réplique déterminée du chef de l'Etat : «S'il y avait confiscation de la politique extérieure par le gouvernement, ce serait un coup $d$ 'Etat »[2]. Cette phrase surprenante est nuancée quelques jours plus tard nuancée [3].

\section{Mars 1986 : la montée en puissance de Matignon}

C'est sur ce fond de cliquetis d'armes, bien plus que sur l'intention sincère de respecter les attributions constitutionnelles de l'autre que la cohabitation s'engage. Pourtant, pendant les premières semaines qui suivent les élections législatives de mars 1986, François Mitterrand croit encore pouvoir s'entendre avec le premier ministre. Il pense le convaincre que la cohabitation dans les affaires internes se déroulerait dans de bonnes conditions si sa prééminence en politique extérieure n'était pas contestée. Les premiers contacts qu'il a avec lui fin mars - début avril - se déroulent dans une atmosphère relativement paisible et le confortent dans son optimisme.

Le choix des ministres des Affaires étrangères et de la Défense nationale se fait sans difficulté majeure mais non sans discussion. Plusieurs noms défilent pour le poste de ministre des Affaires étrangères. L'accord se fait finalement sur Jean-Bernard Raimond. Cet ancien conseiller de Georges Pompidou a gardé entre 1981 et 1986 de bons rapports avec les dirigeants socialistes. C'est François Mitterrand qui en 1985 l'a nommé ambassadeur à Moscou. L'entente est plus rapidement trouvée pour le poste de ministre de la Défense sur la personne d'André Giraud, préféré, et de loin, à François Léotard.

Quelques règles du jeu sont rapidement établies. Le Président recevra toutes les semaines ces deux ministres et tout haut fonctionnaire qu'il demandera à voir. Notes et télégrammes diplomatiques du Quai d'Orsay continueront à affluer normalement. Le déclenchement de l'arme nucléaire restera une prérogative du chef de l'Etat. Au cours d'une réunion à l'Elysée, le 2 avril, Jacques Chirac et André Giraud reconnaissent également explicitement la "prééminence » du chef de l'Etat en ce qui concerne les opérations militaires au Tchad et s'abstiendront de toute remise en cause de la doctrine du $16^{\mathrm{c}}$ parallèle critiquée par le RPR avant son accession au pouvoir. De même que sera reconnu au Président son droit de grâcier les terroristes arrêtés et condamnés en France. Inversement, quand le premier 
ministre demande au Président d'assister au sommet des pays industrialisés qui doit se tenir à Tokyo en avril, ce dernier n'y fait pas obstacle.

Ces premiers contacts donnent au Président l'impression que le gouvernement agira avec modération à son égard. Mais la sérénité présidentielle sera vite écourtée. Le premier ministre s'écarte assez rapidement de l'esprit des négociations préliminaires. Le chef de l'Etat prend progressivement conscience des menaces qui pèsent sur sa fonction. Il lui apparaît que le dessein du premier ministre n'est pas simplement d'acquérir la stature internationale indispensable à un futur candidat à l'élection présidentielle ou de redonner vie aux articles 20 et 21 de la Constitution, dans le respect des prérogatives constitutionnelles du chef de l'Etat, mais de le marginaliser, de le « Renécotyser ». Non pas brutalement ou par une action provocatrice, mais par un accaparement progressif des affaires, une opération de « désertification » de l'Elysée consistant à réduire l'information présidentielle, à détourner l'attention des gouvernements étrangers vers Matignon. Seule la dimension jupitérienne du pouvoir présidentiel - l'emploi de la force armée - n'est pas convoitée. Aucune décision importante concernant les opérations extérieures n'est prise sans l'aval du chef de l'Etat : les décisions concernant les forces françaises agissant au Liban sous couvert de l'ONU, le soutien français à Hissène Habré, de même que l'envoi au Togo, fin septembre 1986, de quelque 200 parachutistes pour aider le général Eyadema à réprimer une rébellion, l'envoi du porte-avion Clémenceau vers le Golfe, seront prises avec le feu vert explicite du Président même quand l'initiative émane du gouvernement. Une seule entorse connue à cette règle : la mise à la disposition de Denis Sassou Nguesso, chef de l'Etat congolais, début septembre 1987, d'un Transall C 130 français aux couleurs à peine maquillées pour l'aider dans une opération de maintien de l'ordre. Cette aide est négociée par Matignon dans le plus grand secret, ce qui vaudra une lettre de rappel à l'ordre du chef de l'Etat au premier ministre.

Partout ailleurs, la prééminence présidentielle est contestée. Le premier ministre veut faire de Matignon le centre d'impulsion et de coordination de la politique étrangère. Entouré d'une équipe d'une douzaine de collaborateurs, il attire tous les dossiers qu'il peut rue de Varenne. Pour le chef de l'Etat, la partie n'est pas facile. L'Elysée est une structure naine. La politique étrangère touche à tous les domaines de l'activité gouvernementale : à l'Economie et aux Anciens combattants, à l'Agriculture, à l'Industrie et au Commerce extérieur. Les ministres ne reconnaissent que l'autorité du premier ministre.

Le Président, qui comptait sur la collaboration étroite des ministres des Affaires étrangères et de la Défense nationale, doit assez rapidement déchanter. Jean-Bernard Raimond et André Giraud se sont très vite positionnés dans le camp du premier ministre. L'information qu'ils fournissent au chef de l'Etat au cours des tête-à-tête qu'ils ont avec lui est soigneusement sélectionnée. Durant ces audiences hebdomadaires, aucune décision d'envergure n'est prise sans que Jacques Chirac en ait été informé au préalable.

Sur plusieurs sujets importants l'information présidentielle se réduit. Ainsi, les télégrammes diplomatiques ne sont pas tous portés à la connaissance de l'Elysée. Le phénomène est signalé à la présidence dès les premiers mois 
par des «fonctionnaires restés loyaux». Le procédé de dissimulation est simple. Depuis le début de la $V^{\mathrm{e}}$ République, l'Elysée est le destinataire d'un jeu complet de télégrammes diplomatiques susceptibles d'intéresser le chef de l'Etat ou ses conseillers. La présidence recevait également toutes les notes de fond élaborées par les services. Parallèlement, le ministre pouvait correspondre par télégramme avec les ambassadeurs sans en informer nécessairement le Président. Ces télégrammes appelés « messages » permettaient à l'ambassadeur de communiquer des informations au ministre seul, ou au ministre et à quelques membres de son cabinet, évitant ainsi leur diffusion dans les bureaux. Un double ne parvient pas d'office à l'Elysée mais seulement quand le ministre le juge utile. Ce canal particulier ne nuisait pas à l'information présidentielle. Les relations entre le Président et le ministre étaient confiantes et aucun responsable du Quai d'Orsay ne se serait aventuré à dissimuler sciemment une information importante au Président.

Sous Roland Dumas, ministre des Affaires étrangères, une modification est apportée, en décembre 1985, à la circulation des messages. Pour éviter tout retard, le ministre a donné instruction au service du chiffre d'adresser d'office à l'Elysée tous les messages concernant les otages et le terrorisme, deux sujets suivis de très près par François Mitterrand.

En 1986, instruction est donnée aux ambassadeurs par Jean-Bernard Raimond et ses collaborateurs de ne plus transmettre de messages à l'Elysée et de faire passer un plus grand nombre de télégrammes sous forme de «distribution message». Ces instructions concernent plus particulièrement trois domaines : la question des otages et les négociations, notamment avec l'Iran et la Syrie ; la lutte contre le terrorisme ; la préparation des voyages du premier ministre et ceux du ministre des Affaires étrangères.

Quand l'Elysée se rend compte de cette manœuvre, une explication s'engage avec Matignon. Le problème a-t-il pour autant été réglé ? Jamais complètement. Sur des sujets sensibles comme celui de la libération du jeune coopérant Pierre-André Albertini, détenu au Ciskeï, le gouvernement va continuer à agir à l'insu de l'Elysée.

D'autres sources d'informations se sont taries ou du moins appauvries. Un des changements les plus sensibles concerne la DGSE (Direction générale de la sécurité extérieure) dont le directeur, le général René Imbot, a, sur ordre du gouvernement, diminué de manière très sensible sa coopération avec l'Elysée.

L'Elysée reçoit beaucoup moins de notes de fond de la part des directions du Quai d'Orsay, y compris les papiers de réflexion et d'analyse. Ces derniers ne remontent plus spontanément à la présidence. Les conseillers de François Mitterrand les reçoivent à leur demande. Encore faut-il qu'ils soient au courant de la production qui s'élabore dans les services du Quai d'Orsay.

D'autres relais indispensables à l'exercice du pouvoir présidentiel ne fonctionnent plus comme auparavant. Les conseillers de l'Elysée ne peuvent plus convoquer des réunions de fonctionnaires et leur passer des instructions. Les multiples réunions qui donnaient aux collaborateurs du chef de l'Etat le pouvoir de tenir le dispositif décisionnel dans un étroit corset ont disparu. Seule la préparation des sommets des pays industrialisés échappe 
véritablement à cette redistribution des tâches. Le sherpa du Président, Jacques Attali, réunit à l'Elysée les hauts fonctionnaires concernés et reçoit toutes les notes qui lui sont nécessaires.

\section{« Le Président a plié, n'a pas rompu... »}

De ce fait, des pans entiers de la politique étrangère - et d'abord ceux qui ont des incidences sur le budget français - vont tomber dans l'escarcelle du gouvernement : la politique de coopération avec les pays du Tiers-Monde, la gestion quotidienne des affaires communautaires, les échanges économiques et financiers bilatéraux, les investissements, les affaires monétaires internationales, les exportations d'armes, l'aide à la francophonie. L'aide française au Tiers-Monde est redéployée au détriment de certains pays d'Amérique centrale, tel le Nicaragua.

Dans les affaires militaires, l'influence du Président s'est également affaiblie. L'initiative revient plus souvent au gouvernement (cela a été vrai en ce qui concerne tant la gestion de la crise tchadienne que l'envoi du porte-avion Clémenceau vers le Golfe) et le Président ne peut prendre de décision d'engagement militaire sans l'approbation du gouvernement dont la collaboration est nécessaire pour la mise en œuvre. Ce qui était autrefois une chasse gardée du Président est devenu un secteur de cogestion obligée.

Les dossiers de politique étrangère liés à la sécurité intérieure (otages, lutte antiterroriste, relations avec l'Iran) passent également sous le contrôle du gouvernement. Hormis la crise aiguë des relations franco-iraniennes de l'été 1987 (qui va aboutir à la rupture des relations diplomatiques et qui a été gérée en commun), ces affaires sont soustraites à l'attention élyséenne qui n'obtiendra généralement qu'une information tardive et partielle. Toutes les tractations en vue de la libération des otages et de la normalisation francoiranienne sont conduites le plus souvent secrètement par le ministère des Affaires étrangères, mais plus encore par celui de l'Intérieur, quand ce n'est pas par un des membres de l'entourage de Jacques Chirac. Les instructions sont données par le premier ministre sans que le chef de l'Etat puisse intervenir.

A de multiples reprises, le chef de l'Etat a contesté que l'incursion de Jacques Chirac dans le «périmètre sacré » ait entraîné un glissement de pouvoirs de l'Elysée vers Matignon. Or, les capacités d'intervention présidentielles ont bel et bien décru. L'initiative appartient autant au Président qu'au premier ministre. La notion de subordination hiérarchique a disparu. L'autorité du Président a été à maintes reprises remise en cause. Sa capacité à faire prévaloir son point de vue, son contrôle sur certains secteurs de la politique étrangère se sont affaiblis.

Pourtant l'offensive chiraquienne n'atteint que partiellement son but. Le chef de l'Etat s'est fait arracher quelques "livres de chair de son pouvoir " [4] mais la machine chiraquienne n'a pas pu le dévorer. "Il a plié, n'a pas rompu, a fini par se redresser ", notera justement Edouard Balladur [5]. François Mitterrand réussit à se préserver une marge d'influence non négligeable, s'appuyant sur le protocole, sur ses collaborateurs, sur des partisans qu'il a gardés un peu partout dans l'administration, l'armée et la police. L'isolement de la présidence est loin d'avoir été systématique. 
En matière de défense, André Giraud a confirmé les dispositions permettant au chef de l'Etat d'accéder à l'information à laquelle il avait déjà accès. A l'état-major des Armées, le général Jean Saulnier, ancien chef d'état-major particulier du Président, continue de se comporter avec la plus grande loyauté vis-à-vis du chef de l'Etat.

Dans les affaires européennes, François Mitterrand a prévu dès avant la cohabitation un dispositif qui lui permette d'être parfaitement à jour des décisions qui s'y préparent. Elizabeth Guigou, conseiller technique à l'Elysée et secrétaire général du Comité interministériel pour les questions de coopération économique européenne (SGCI), Jacques Delors à la présidence de la Commission européenne et François Scheer, représentant de la France auprès des Communautés européennes et ancien directeur de cabinet de Claude Cheysson, veilleront à la bonne information du chef de l'Etat. Certes, la gestion des affaires communautaires est réservée au premier ministre, mais Jacques Chirac doit composer avec le Président pour tous les dossiers discutés en Conseil européen. Aussi chacun de ces Conseils est-il précédé d'une réunion à l'Elysée où le chef de l'Etat et les membres du gouvernement harmonisent leurs positions.

Dans les affaires africaines, les ponts sont loin d'être coupés entre l'Elysée et les représentants français dont la plupart entretiennent des relations avec les deux centres de pouvoir. Après un éloignement de douze ans des centres de décision, Jacques Foccart à Matignon régularise à nouveau les relations politiques et économiques avec l'Afrique. Celui-ci laisse parvenir à la rue du Faubourg Saint-Honoré une information abondante. Le Président et ses collaborateurs entretiennent avec la plupart des chefs d'Etats africains un contact régulier. D'ailleurs aucun pays n'a voulu suivre les conseils de Matignon et prendre le risque de se couper de François Mitterrand qui demeure un interlocuteur de premier plan pour les grandes questions politiques. Aux grands sommets (sommet des pays industrialisés, Conseil européen, sommet franco-africain), c'est lui qui est accueilli comme chef de la représentation française et Jacques Chirac comme son second. Pour se démarquer du Président, le premier ministre sera amené à organiser ses propres voyages et s'emploiera à leur conférer le faste auquel un chef d'Etat a droit.

Plus frappant encore est le fait que François Mitterrand ait réussi à dissuader Jacques Chirac de remettre en cause certaines de ses orientations de politique étrangère. En avril 1986, Jacques Chirac confiait à un de ses proches conseillers : "Certes, il existe en France un consensus en politique étrangère. Mais je modifierai quelques orientations ". Et le premier ministre de mentionner parmi les modifications souhaitées l'attitude de la France visà-vis de l'Initiative de défense stratégique (IDS) proposée par Ronald Reagan, la politique française à l'égard du Nicaragua, de la Turquie et de l'Afrique du Sud, les relations avec l'Iran, enfin la loi de programmation militaire votée en 1983 qu'il a l'intention de faire abroger pour en proposer une nouvelle.

Jacques Chirac devra se contenter de modifications mineures. François Mitterrand laisse s'engager l'amélioration des relations franco-turques, symbolisée par la visite d'André Giraud à Ankara les 23 et 24 mai 1986. Elle ne constitue pas une innovation majeure ; le ministre de la Défense a, 
plusieurs mois auparavant, été précédé par Etienne Manac'h, ancien ambassadeur de France en Chine, à la demande de François Mitterrand. Jacques Chirac obtient, le 9 mai 1986, le retour à Pretoria de l'ambassadeur français rappelé à Paris par Laurent Fabius en juillet 1985, mais cette décision n'a pas, elle non plus, rencontré l'opposition de François Mitterrand. La mesure demandée par Laurent Fabius avait, alors, été acceptée du bout des lèvres par le chef de l'Etat. Le processus de normalisation des relations francoiraniennes ne constitue pas non plus une innovation importante, lorsque l'on sait que François Mitterrand et Roland Dumas s'étaient déjà engagés dans cette voie, même si leur action ne fut couronnée d'aucun succès. S'il existe des changements, ils portent sur la tactique et les moyens plutôt que sur le principe de normalisation. François Mitterrand a marqué une préférence pour une négociation visant à la libération de l'ensemble des otages plutôt qu'au "saucissonnage " du processus qui conduit à libérer les otages par paquets de deux. De même qu'il n'était pas d'accord avec la méthode du troc, consistant, notamment, à expulser des Moudjahiddin en échange d'une libération d'otages.

Dès avant le 16 mars 1986, le RPR avait également affiché son intention de renégocier certaines clauses du traité d'adhésion de l'Espagne et du Portugal à la Communauté européenne. Mais Jacques Chirac se heurtera à un refus net du chef de l'Etat se fondant sur l'article 52 de la Constitution qui lui attribue le pouvoir de négocier et de ratifier les traités.

Mais c'est dans les affaires de défense que François Mitterrand impose le mieux sa prééminence, contredisant sa promesse de respecter "la Constitution, toute la Constitution, rien que la Constitution". Jacques Chirac fait abroger la loi de programmation militaire des socialistes et fait voter, avec l'appui de ces derniers, une nouvelle loi prévoyant un effort financier plus important et plus régulier, mais il n'arrive pas à imposer au Président les autres changements souhaités. Ses idées sur l'IDS seront purement et simplement rejetées. (Il y renoncera d'ailleurs lui-même plus tard.) Le Président obtient confirmation de la priorité à la modernisation des sousmarins nucléaires alors que le gouvernement voulait avantager la mise sur pied d'un missile terrestre mobile, dit "à roulettes ». François Mitterrand réussit à faire en sorte qu'aucune décision ne soit prise en ce qui concerne le missile mobile. La loi de programmation propose simplement de préparer la mise au point d'un missile balistique léger. Mais la quantité et le mode de déploiement de ces missiles seront à définir en 1988-1989. Demi-échec seulement, rétorquera le gouvernement : la loi ne prévoit pas l'abandon pur et simple des « roulettes ».

Quant à la doctrine d'emploi de l'arme nucléaire tactique, François Mitterrand met un terme à la controverse par ses déclarations au Camp de Caylus, le 13 octobre 1986. Le 12 septembre 1986, devant les auditeurs de l'Institut des hautes études de défense nationale, Jacques Chirac laissait entendre que l'arme nucléaire tactique pourrait être utilisée autrement qu'en une frappe unique et pas nécessairement comme arme d'" ultime avertissement » avant le passage à la frappe stratégique. L'arme nucléaire tactique est conçue comme un moyen de remporter une victoire militaire : "Cet avertissement aura pour objectif non seulement d'adresser un signal sans équivoque à l'agresseur, mais aussi d'enrayer la dynamique de l'agression". François Mitterrand répliquera sèchement en réaffirmant que "ces armes ne 
sont pas séparables de la stratégie ". L'annexe de la loi de programmation militaire montre que sur ce point Jacques Chirac n'a pas voulu engager une épreuve de force avec le Président puisqu'elle rétablit la notion "d'ultime avertissement » et l'appellation d'«arme nucléaire préstratégique » chère au Président.

Le gouvernement cherchera, ici encore, à minimiser en privé la portée des propos du Président. "Il s'agit de rhétorique", ont dit en substance les proches du premier ministre, «or, ce sont les décisions concrètes qui comptent». En matière stratégique, la rhétorique atomique peut s'avérer dissuasive. En matière cohabitationniste, elle peut être meurtrière : celui qui la formule est perçu comme le responsable suprême de la défense. Or, en adoptant face au Président un profil bas - sur ces questions comme sur celles de la deuxième option zéro et de la modernisation des armes nucléaires tactiques de l'OTAN, sujets sur lesquels il avait émis une opinion différente de celle du Président -, Jacques Chirac accorde un avantage décisif à ce dernier : ce sont les propos du chef de l'Etat et non les siens qui traduisent la position officielle de la France.

«Nous avions tort de ne pas nous opposer plus nettement à $M$. Mitterrand sur les questions de défense nucléaire où il faisait preuve de trop de compréhension envers les accords américano-soviétiques concernant la réduction des armements ", dira plus tard Edouard Balladur non sans candeur [6]. Le gouvernement a commis une erreur de tactique en laissant au chef de l'Etat le pouvoir de déclencher l'arme nucléaire s'imaginant faire une concession peu coûteuse. Il était incohérent de reconnaître au Président le privilège du feu atomique et lui contester le droit d'élaborer la doctrine et de choisir les armes. Il y avait une faille dans laquelle François Mitterrand n'a pas manqué de s'engouffrer. Il s'est investi non seulement d'un droit de veto mais aussi d'une capacité d'initiative qu'il n'a nulle part ailleurs. Il négocie secrètement avec le chancelier Kohl la mise sur pied d'un Conseil de défense franco-allemand et l'annonce publiquement sans en informer au préalable Jacques Chirac. Aussi bien sur l'affaire du démantèlement des euromissiles que dans la négociation interne à l'OTAN sur la modernisation des armes nucléaires tactiques, il réagit vite, prenant de court le gouvernement et étouffant tout débat au sommet de l'Etat.

\section{Les trois sphères d'influence}

Au terme des neuf premiers mois de cohabitation, il s'est ainsi établi un partage de la politique étrangère en trois sphères d'influence : une sphère à prédominance présidentielle : la défense, les relations franco-allemandes, le désarmement; une sphère à prédominance gouvernementale : l'Afrique et la coopération, le Proche-Orient dans sa dimension sécurité intérieure (otages, terrorisme, Iran), les relations économiques, commerciales et financières avec les pays étrangers (les négociations de gaz algérien, naguère sous contrôle de François Mitterrand, ont été arbitrées, côté français, par Jacques Chirac). Entre les deux subsiste un important domaine de cogestion où aucune prééminence n'apparaît clairement, où l'un ne peut agir sans l'accord de l'autre. Entrent dans cette catégorie, notamment, les opérations militaires, les grandes décisions en matière communautaire, l'attitude à l'égard du conflit israélo-arabe. 
Ce partage n'est inscrit nulle part dans la Constitution. Il la contredit même dans une certaine mesure. En pratique le Président impose mieux son autorité en politique de défense qu'en politique étrangère alors que la Constitution prévoit le contraire : un partage équitable entre le chef de l'Etat et le premier ministre en matière de défense et des prérogatives plus nettement précisées en faveur de l'hôte de l'Elysée en diplomatie.

François Mitterrand et Jacques Chirac sont tous les deux partis avec l'idée de faire le moins de concessions possible. En deux ans, que d'espoirs déçus. La cohabitation les a obligés à des effacements alternés. C'est le résultat des élections législatives du 16 mars 1986 qui a fixé dès le début les limites des ambitions. La combativité de l'un et de l'autre dépend des chances de sortir vainqueur de cette partie de bras de fer sans créer une crise politique qui bénéficierait à Raymond Barre.

Mais, des deux, c'est le premier ministre qui redoute le plus le dérapage. Jacques Chirac, a-t-on dit souvent, ne veut pas paraître s'en prendre à une institution mise en place par le général de Gaulle. Ce ne sera pas là une de ses moindres contradictions. Sa volonté d'apparaître comme le responsable suprême de la diplomatie l'amènera malgré tout à œuvrer pour l'abaissement de la présidence qu'il convoite. Jacques Chirac s'étend, occupe le terrain, mais, quand il sent que le Président ne va pas céder, il bat en retraite. Car la diplomatie et la défense sont secondaires dans le projet RPR-UDF par rapport à l'économie et au social. Le gouvernement a un besoin vital de temps pour démontrer sa capacité à redresser la situation économique.

Installé dans une popularité que les sondages ne cessent de confirmer, le Président, lui, redoute moins la crise politique. La politique étrangère représente, pour lui, un enjeu vital. Abandonner le terrain, c'est disparaître quasiment comme agent actif de la scène politique. Mais il ne peut la provoquer pour un motif qui apparaîtrait futile. Comme le nucléaire, l'arme est trop lourde et ne peut être utilisée qu'en dernier recours. Il ne va pas ouvrir une crise tous les jours pour manquement au respect de la prééminence présidentielle. Il est un peu piégé par cette Constitution qui propose aux Français une dyarchie. Il ne pourra pas faire autrement que céder du terrain. Il va éviter de se battre dans les domaines où il a peu de prise ou qui ne présentent qu'un faible intérêt dans le débat politique français, pour s'investir dans ceux qui peuvent être considérés comme «vitaux pour les intérêts de la France».

Selon la Constitution, ou l'interprétation qu'il en donne, François Mitterrand a souvent répété qu'il était en charge de la "sécurité extérieure et intérieure " de la France. Or, on ne peut manquer d'être frappé par son effacement lors des attentats sanglants de septembre 1986. Il maintient son voyage prévu en Indonésie, permettant au premier ministre de renforcer son image de premier responsable de la politique de la France. C'est que le Président, tout comme le premier ministre, sait faire preuve de pragmatisme et calculer au mieux les risques politiques. François Mitterrand a laissé le premier ministre se mettre en avant dans un domaine où les risques d'échecs ne sont pas négligeables. Mais il y a plus. Le Président n'est pas à l'aise dans cette affaire de lutte contre le terrorisme. Le gouvernement entend employer des moyens que le Président ne veut ni cautionner ni 
réprouver. Les socialistes dans ce domaine n'ont pas réalisé leur propre synthèse entre les contraintes de sécurité et le respect des droits de l'homme. Le gouvernement socialiste n'avait pas su gérer cette contradiction. De ce fait, le Président a laissé le champ complètement libre à Jacques Chirac.

Inversement, s'opposer au gouvernement sur les questions stratégiques ne présente que des avantages pour le Président. La sacralisation du pouvoir atomique du Président est fortement ancrée dans l'opinion. En cas de désaccord, il n'a aucun mal à contraindre Jacques Chirac à choisir entre la crise et l'effacement. Quand enfin ils s'entendent et trouvent avantage à collaborer, comme pour le Liban, le Tchad, lors de la crise du Golfe ou pour interdire aux avions américains le survol du territoire français, on les verra travailler ensemble étroitement, discrètement.

Le Président et le premier ministre paraderont jusqu'au bout chacun de son côté avec des allures monarchiques mais éviteront de se déchirer trop ouvertement. C'est à mettre à leur actif.

\section{Les virtualités de conflit}

La sagesse et la modération l'ont emporté lors des moments difficiles. Aucun des ennemis avérés de la France n'a eu à profiter de la rivalité entre les deux hommes : ni Kadhafi, qui aurait souhaité que les dirigeants français règlent leurs propres querelles plutôt que de s'immiscer dans celles du continent africain; ni l'OJR (l'Organisation de la justice révolutionnaire) qui en représailles aux propos sévères de François Mitterrand menaçait de tuer un des otages, Jean-Louis Normandin, si Jacques Chirac ne «clarifiait » pas les propos du Président [7]. "Le gouvernement, répondit le porteparole du premier ministre, entend poursuivre avec ténacité et fermeté sa lutte contre le terrorisme d'où qu'il vienne et sous quelque forme qu'il se manifeste ».

La cohabitation en politique étrangère n'en a pas moins été une lutte pour l'hégémonie, entrecoupée de moments d'entente, voire de réelle connivence. Elle a été une bataille souterraine, feutrée, où chacun des adversaires a agi sans brutalité, mais par feintes successives. Les relations entre la présidence et le gouvernement se sont déroulées dans un climat de méfiance endémique. La cohabitation a créé une atmosphère malsaine de dissimulation et de dénigrement. Dans certains secteurs (Est-Ouest, relations franco-allemandes, Proche-Orient), une diplomatie double, concurrente, s'est installée, entraînant un énorme gaspillage d'énergie. Les deux ont passé un temps considérable à se cacher des informations ou à tenter de compléter celles qui leur échappaient.

La diplomatie française a perdu de sa liberté d'initiative et de son imagination. Celle-ci s'est investie dans la communication, dans des opérations valorisantes pour l'image de chacun des deux grands protagonistes. Il est bien difficile de développer une initiative un tant soit peu hardie dans une période jugée provisoire où l'essentiel consiste à ne pas faire de gaffe ou à savoir récupérer à son avantage les idées des autres.

Certains problèmes qui méritaient d'être mieux posés sur la place publique ont été étouffés ou obscurcis (les prises de position sur le rôle futur de la 
France dans la défense de l'Europe constituent un bon exemple). Les véritables intentions de l'un ou de l'autre sont difficiles à saisir et obligent à se fier aux supputations d'experts extérieurs au gouvernement. Raymond Barre porte une part de responsabilité dans cette situation. Guetteur ironique des discordances entre le Président et le premier ministre, il les a souvent incités à une prudente solidarité de façade.

On a souvent affirmé que la cohabitation en France a été possible grâce au « consensus ». C'est inexact. Les désaccords ont été nombreux, sur la défense, le désarmement, l'Afrique du Sud, l'attitude à l'égard de certains Etats du Tiers-Monde. Mais aucune de ces divergences n'avait un caractère passionnel, n'appelait des révisions déchirantes. Même si certains experts pensaient que des choix décisifs devaient être faits en matière de défense, ni l'opinion, ni les partis politiques ne les ont vécus de manière dramatique.

La Constitution est encore loin d'avoir prouvé son «adaptation aux conjonctures de crise ». Nous n'avons connu jusqu'ici qu'une seule expérience de la cohabitation, et celle-ci s'est déroulée dans un contexte comportant un grand nombre d'éléments modérateurs et dans une conjoncture diplomatique paisible pour la France.

Avec ses ambiguïtés, la Constitution porte en elle d'importantes virtualités de conflit. Dans un contexte politique avantageux pour le gouvernement, on voit mal ce qui empêcherait ce dernier de chercher à en découdre avec le Président et de l'obliger à choisir entre la résignation et la démission. Il pourrait fort bien le déposséder de cet atout majeur que constitue le pouvoir de déclencher l'armement nucléaire, pouvoir reposant jusqu'ici sur un décret - pas même une loi - du 14 janvier 1964 , qui ne concerne d'ailleurs que l'engagement des forces aériennes stratégiques. Les autres composantes, sous-marine et terrestre, de la force de dissuasion nucléaire n'étaient pas encore mises sur pied. Selon Jacques Fournier, secrétaire général du gouvernement de 1982 à 1986, on peut «concevoir qu'un gouvernement issu d'une majorité hostile au Président mette en chantier une réforme destinée à limiter ses pouvoirs en matière de défense. Celle-ci aurait pu prendre la forme d'une loi relative aux "principes fondamentaux de l'organisation générale de la défense nationale », matière confiée au législateur par l'article 34 de la Constitution » [8]. L'hôte de l'Elysée pourrait alors se demander si, dans les années 1986-1988, le Président n'a pas mangé son pain blanc. A moins que d'ici là un aménagement de la Constitution n'intervienne, la classe politique tirant enfin les leçons de la cohabitation. Etrangement, aucun des deux grands acteurs ne s'est vraiment livré à une analyse rétrospective de ces événements, feignant de croire qu'il ne s'est rien passé. Estimeraient-ils tous les deux ne rien avoir à gagner à l'évocation de ce passé récent?

\section{La revanche de l'Elysée}

S'il est un mérite que l'on ne peut contester à la cohabitation, c'est celui d'avoir incité le chef de l'Etat à préciser ses conceptions quant à l'avenir de la fonction présidentielle. Réflexion qui n'est pas dénuée d'intérêt. François Mitterrand réaffirme son rôle de "responsable de la route à suivre par la Nation quand sa sécurité et sa place dans le monde sont en jeu, responsable 
des grandes orientations de la politique extérieure et de la défense du pays »[9]. Néanmoins, le chef de l'Etat rejette explicitement la conception d'un «président commande-tout» [10] qui a été celle de la $V^{\mathrm{e}}$ République : «J'observerai (...) que le Président n'a pas à gouverner, mais à définir les grands choix »[11]. François Mitterrand ne manquera pas d'évoquer la «trace» «profonde» que la cohabitation laissera pour affirmer que «chacun des pouvoirs sait désormais qu'il existe et voudra exercer sa pleine compétence y compris lorsque majorité parlementaire et majorité présidentielle coïncideront à nouveau » [12].

L'affirmation de la «pleine compétence de chacun des pouvoirs » et le rejet d'une présidence «commande-tout » laissent supposer que le premier ministre (voire le Parlement) aurait après le 8 mai 1988 un rôle important à jouer dans la conduite de la diplomatie et la défense. Cette conception a-telle prévalu? Michel Rocard exerce-t-il la «pleine compétence » qui lui est dévolue par la Constitution? Joue-t-il au moins un rôle plus important que ses prédécesseurs socialistes Pierre Mauroy et Laurent Fabius ? La cohabitation aurait-elle accouché d'un « nouveau Président »? [13].

Les événements qui se sont succédé depuis mai 1988 montrent que non seulement aucun rééquilibrage n'a eu lieu mais que le retour à l'unité du système de décision s'est accompagné d'un renforcement du poids du Président par rapport aux années 1981-1986. Jamais la prééminence de François Mitterrand n'a été aussi forte. Le Président ne se contente pas de "définir les grands choix». Il gouverne, tranche souvent jusque dans le détail (visite de Yasser Arafat à Paris, secours apporté aux chrétiens et aux musulmans du Liban, relations avec les pays de l'Est, dissuasion nucléaire, effacement de la dette des pays les plus pauvres, envoi en août 1989 d'une partie de la flotte française à destination des côtes libanaises).

Tout semble montrer de la part du chef de l'Etat un souci de voir le premier ministre regagner sa place, d'effacer toute trace de la cohabitation, de reprendre entièrement le contrôle de la politique étrangère et militaire. Le premier ministre n'assistera plus aux réunions au sommet, le ministre des Affaires étrangères reprenant sa place aux côtés du chef de l'Etat. Le premier ministre retrouve son rôle traditionnel, légèrement retouché, de sous-traitant de haut niveau pour le compte du président de la République. $\mathrm{La} \mathrm{V}^{\mathrm{c}}$ République voue le premier ministre à un rôle d'appui à la politique conduite par le chef de l'Etat, dans les secteurs situés à la charnière de la diplomatie et de la politique économique : arbitrages budgétaires qui n'appellent pas l'intervention du Président (coopération, questions économiques européennes, budget de la défense) ; renforcement de la présence économique, commerciale et technologique de la France à l'étranger ; règlement de litiges délicats avec certains partenaires de la France ; contrôle des exportations des produits stratégiques sensibles (à l'exclusion de matériels nucléaires sur lesquels l'Elysée se réserve un droit de regard). Matignon réalise ainsi une œuvre non négligeable de préparation, de coordination et d'arbitrage qu'aucun autre ministère ne peut effectuer et que l'Elysée ne peut accomplir faute d'effectifs suffisants.

Michel Rocard et son équipe remplissent leur mission en concertation étroite avec la présidence de la République et dans le respect des orientations du chef de l'Etat. Le nouveau premier ministre n'aspire pas à jouer 
un rôle important dans la définition de la politique étrangère, à concurrencer le Président ou à lui porter ombrage. Certes, quelques-unes de ses déclarations ont pu laisser penser le contraire. Notamment celle portant sur l'avion de combat Rafale. Il évoquait à ce sujet son état de "sinistre avancé ", laissant ainsi douter de la volonté du gouvernement français de poursuivre le programme, alors que Jean-Pierre Chevènement, le ministre de la Défense, avait exclu cette hypothèse. Mais le premier ministre est revenu sur cette déclaration mettant un terme à toute spéculation. De même, on ne pouvait manquer de s'interroger sur l'entretien accordé à L'Expansion, dans lequel il exprimait ses réserves concernant l'harmonisation de la TVA européenne. Propos en contradiction avec les engagements de François Mitterrand dans sa «Lettre à tous les Français ». A noter également cette phrase prononcée le 18 août 1989 à la suite de la décision du Président d'envoyer la flotte française au large de Beyrouth : "Il nous faudra imposer le silence localisé des canons, le temps des opérations humanitaires. La seule présence de nos navires devrait y suffire ". François Mitterrand a dû rectifier ce propos musclé par un langage plus apaisant.

Tous les circuits de décision que Jacques Chirac et son gouvernement avaient, non sans une certaine délectation, déconnectés de l'Elysée pour les brancher sur l'hôtel Matignon ont été remis en l'état. Les fils aboutissent à nouveau tous directement au Président ou à ses collaborateurs. Le Président a renoué avec la tradition du dialogue privilégié avec son ministre des Affaires étrangères redevenu comme jadis le "bras séculier » du Président. A un niveau juste inférieur, tous les grands secteurs sont suivis et coordonnés par les conseillers du chef de l'Etat qui tiennent ce dernier informé et transmettent aux plus hauts responsables des ministères les instructions présidentielles : les affaires européennes sont suivies par Elizabeth Guigou, conseillère du Président et secrétaire général du SGCI ; Claude Arnaud, diplomate de haut rang et proche du chef de l'Etat, supervise avec JeanChristophe Mitterrand les affaires africaines et malgaches. Toutes les semaines il préside dans son bureau une réunion en présence des principaux responsables de ce secteur; la préparation des sommets des pays industrialisés relève toujours de la responsabilité de Jacques Attali qui peut en outre se voir confier par le Président tout autre dossier; les affaires stratégiques et de désarmement sont traitées par Hubert Vedrine, également porteparole de la présidence; les affaires de renseignement font l'objet d'une surveillance tout à la fois de la part du secrétaire général de l'Elysée, JeanLouis Bianco, du directeur de cabinet, Gilles Ménage, et du chef de l'étatmajor particulier, l'amiral Jacques Lanxade, qui assure en même temps le lien entre le Président et l'état-major des Armées.

Une réunion domine peut-être toutes les autres par son importance : celle que préside tous les vendredis Jean-Louis Bianco et à laquelle assistent les membres de l'équipe élyséenne (Jacques Attali, l'amiral Lanxade, Christian Sautter, secrétaire général adjoint de la présidence, Hubert Vedrine, Elizabeth Guigou, Jean Musitelli, conseiller diplomatique, aujourd'hui remplacé par Loïc Hennekin), deux collaborateurs du premier ministre (Philippe Petit et Dominique Perreau), le directeur de cabinet du ministre des Affaires étrangères et le secrétaire général du Quai d'Orsay. Mis sur pied longtemps avant mars 1986, ce mini-conseil des Affaires extérieures avait, à la demande de Jacques Chirac, cessé de se réunir pendant la cohabitation. Il est ressuscité après mai 1988 pour jouer un rôle accru dans la régulation de 
la politique étrangère : échange d'information, coordination, délibération, mise au point de certaines décisions.

Il n'est quasiment pas d'initiative importante depuis mai 1988 qui n'ait émané du Président. Il n'en reste pas moins qu'à plusieurs reprises Michel Rocard a eu un rôle diplomatique personnel à jouer : c'est à son initiative que la France a entrepris une amélioration des relations avec la NouvelleZélande et l'Australie, relations passablement dégradées notamment depuis l'affaire Greenpeace. C'est lui qui a désamorcé la crise de la pêche avec le Canada. C'est surtout à son instigation que Matignon a organisé la conférence de La Haye sur la protection de l'environnement. Certes, il ne s'agit pas d'initiatives spectaculaires et ces interventions du premier ministre ne revêtent pas un caractère nouveau.

Tous les présidents de la $V^{e}$ République ont encouragé leur premier ministre à exercer un rôle intuitu personae limité. Insignifiant au temps des présidences de Gaulle et Pompidou, ce rôle s'est accru à partir de la présidence de Valéry Giscard d'Estaing. Ce dernier avait laissé Jacques Chirac se passionner pour l'Irak et donner une impulsion importante aux rapports avec ce pays. De Raymond Barre, il avait fait un de ses conseillers privilégiés pour les questions économiques et monétaires européennes. Mais c'est Pierre Mauroy qui fut le plus impliqué dans la diplomatie, orchestrant de Matignon la réorientation de la politique française à l'égard de l'Amérique centrale, nouant des relations étroites avec Raoul Alfonsin bien avant son accession à la magistrature suprême. Outre les missions qu'il s'est vu confier, notamment au Gabon et au Tchad, il a contribué à régler le contentieux franco-algérien ouvrant la voie à une rencontre Chadli-Mitterrand en novembre 1983. Laurent Fabius, quant à lui, s'est montré particulièrement attaché à combattre l'apartheid en Afrique du Sud et s'est intéressé tout particulièrement aux relations avec le Japon, Israël et la Corée du Sud. Mais au total, son passage à Matignon a moins compté que celui de Pierre Mauroy. Certes, son séjour rue de Varenne fut de plus courte durée, mais là n'est pas la raison principale. Pierre Mauroy a bénéficié d'un contexte particulier marqué par la présence à l'Elysée d'un Président soucieux au début de son septennat de donner une image plus démocratique de la gestion de la diplomatie, de lui conférer un caractère moins centralisateur. Mais ce genre d'initiatives que prennent les premiers ministres se comptent généralement sur les doigts d'une main et ne contribuent que modestement au façonnement de la politique étrangère.

Il faut toutefois souligner qu'à la différence de ses prédécesseurs socialistes, Michel Rocard porte pour les questions de renseignement un intérêt marqué. Constatant les lacunes dans l'animation et la coordination des services spéciaux, Michel Rocard a obtenu la réactivation du Comité interministériel du renseignement. Le CIR (organisme créé en 1962) regroupe, sous la présidence de Michel Rocard, tous les ministres concernés par les questions de sécurité intérieure et extérieure. Il se réunit deux fois par an et a pour objet l'élaboration d'un plan national du renseignement. Michel Rocard a également obtenu la mise sur pied d'un Comité permanent du renseignement. Présidé par Jean-Paul Huchon, son directeur de cabinet, le CPR réunit une fois par mois, environ, tous les responsables des services spéciaux ainsi que les directeurs de cabinet des ministres des Affaires étrangères, de la Défense nationale et de l'Intérieur. C'est à ce niveau que 
l'action du premier ministre est la plus efficace. Le CPR examine les plans de renseignements des différents services, discute de toutes les grandes menaces du moment. Il a ainsi, entre autres questions, traité des risques de transfert de technologie aux pays de l'Est, de la menace islamique, des mouvements autonomistes, des risques qu'il y avait à tenir le sommet des pays industrialisés à Paris au moment des festivités du bicentenaire de la Révolution. Michel Rocard a, en outre, nommé à son cabinet un expert en matière de renseignement en la personne de Remy Pautrat, ancien patron de la DST (Direction de la sécurité du territoire). Mais, ici également, il n'y a pas de grande initiative prise sans l'accord du président de la République. Les décisions essentielles ainsi que la supervision des activités majeures des services de renseignements continuent à relever du chef de l'Etat.

\section{L'insuffisance de contrepoids}

Que François Mitterrand n'ait pas souhaité déroger à ces habitudes contrairement aux voux formulés pendant la cohabitation peut prêter à la critique, mais ne peut véritablement étonner. François Mitterrand a acquis au cours de son premier septennat à la fois une expérience et une vision des relations internationales qui laissent peu de place à des influences extérieures. Sa stature internationale lui permet d'en imposer facilement aux membres du gouvernement. Au sommet de l'Etat, lui seul est resté sept ans durant au centre de la politique étrangère de la France, même si la cohabitation a soustrait de son regard certains dossiers épineux comme celui des otages. Dans le domaine de la défense, son avantage est encore plus grand car, même pendant les années 1986-1988, il n'a cessé de les dominer. Pendant ces sept ans, les titulaires de tous les grands postes de responsabilité ont changé. Aux Affaires étrangères quatre ministres se sont succédé (Claude Cheysson, Roland Dumas, Jean-Bernard Raimond et à nouveau Roland Dumas). A la Défense, quatre ministres également se sont succédé (Charles Hernu, Paul Quilès, André Giraud et Jean-Pierre Chevènement) ainsi que trois chefs d'état-major des Armées (les généraux Jannou Lacaze, Jean Saulnier et Maurice Schmitt).

L'avantage de la durée a également joué pour les collaborateurs personnels du Président. En mai 1988, les entourages ministériels qui se forment à Matignon et aux Affaires étrangères sont composés de fonctionnaires qui, pour la plupart, étaient, pendant la cohabitation, restés éloignés des centres de décision. L'équipe élyséenne, elle, a traversé cette même période aux premières loges. Avant même l'élection présidentielle, elle a entrepris à la demande du Président une réflexion sur les moyens de relancer la politique étrangère, sur les initiatives que François Mitterrand pourrait prendre après son élection, les hommes sur lesquels il pourrait s'appuyer. Lors du démarrage du second septennat, les conseillers du Président vont jouer de ce fait un rôle prépondérant d'orientation et d'impulsion.

A ces pesanteurs s'en ajoute une plus importante : celle des engagements du candidat à l'élection présidentielle, élection majeure de la $\mathbf{V}^{\mathrm{e}}$ République. Un président élu ne peut accepter que le premier ministre qu'il nomme vienne entraver les orientations qu'il a promis de respecter. Comment, dès lors, un premier ministre ou un ministre des Affaires étrangères 
peut-il exister par lui-même, prendre un tant soit peu d'autonomie ? Tout ceci a entraîné une tutelle accrue de l'Elysée sur l'appareil diplomatique qui va jusqu'au contrôle de tout communiqué émanant du Quai d'Orsay. Les hauts responsables du ministère des Affaires étrangères ne cachent pas que leur liberté d'initiative est des plus limitées et que tout écart par rapport aux directives présidentielles peut entraîner un rappel à l'ordre.

Après deux années de cohabitation, le Président se retrouve à nouveau maître incontesté de la politique étrangère. Ce retour du pendule a inspiré bien des propos sur l'absolutisme de la monarchie présidentielle. Plusieurs exemples montrent pourtant que ces craintes sont loin d'être fondées, que l'on est loin du pur arbitraire : le chef de l'Etat s'entoure d'avis, même s'il lui arrive, comme à ses prédécesseurs, de décider seul sans consultation préalable, lors de ses rencontres en tête-à-tête avec ses homologues étrangers.

Si danger il y a, il tient plutôt à l'insuffisance autour du Président de véritables contrepoids. L'opposition n'a jamais été aussi faible et divisée. La prééminence intellectuelle du Président sur ses ministres et conseillers n'a jamais été aussi marquée depuis mai 1981. L'organisation qui entoure le Président manque de quelques personnalités fortes capables de contester certains de ses choix au risque de l'indisposer. Indiscutablement, Claude Cheysson remplissait ce rôle. Roland Dumas qui dispose de la confiance absolue de François Mitterrand incline plus à s'adapter au Président qu'à s'opposer à lui.

Le temps passé à l'Elysée est, pour tout Président, un atout et une menace. Il accroît son expertise et son autorité, mais éloigne de lui les indispensables trublions qui l'empêcheront de trop se fier à ses certitudes. 


\section{NOTES BIBLIOGRAPHIQUES}

[1] Conférence de presse, 21 juillet 1986.

[2] Déclaration faite dans le Vercors, le 10 juillet 1985.

[3] Déclaration faite au cours d'une prestation télévisée, le 14 juillet 1985.

[4] Au cours d'une interview accordée à Jean Lacouture, François Mitterrand ironise sur les nouveaux dirigeants de 1986 convaincus " qu'ils m'arrachaient livre de chair après livre de chair de mon pouvoir ", L'Express, 4 septembre 1987.

[5] Edouard Balladur, Passion et longueur de temps, Fayard, Paris, 1989, p. 224.

[6] lbid., p. 204.

[7] Après le verdict condamnant Georges Ibrahim Abdallah à perpétuité, François Mitterrand déclarait qu'il ne faut "pas traiter avec les terroristes" ni "échanger un criminel contre un innocent $»$.

[8] Jacques Fournier, Le travail gouvernemental, Presses de la Fondation nationale des sciences politiques et Dalloz, Paris, 1987, p. 75.

[9] «Lettre à tous les Français ».

[10] Entretien accordé à la revue Pouvoirs, 1988, n" 45.

[11] « Lettre à tous les Français ».

[12] Pouvoirs, op. cit.

[13] Titre du livre d’Olivier Duhamel et Jérôme Jaffré, Le Seuil, Paris, 1988. 